\section{ADMINISTRACIÓN PÚBLICA}

\section{resumen}

El documento que se presenta parte del reconocimiento de las instituciones como representaciones mentales que le son útiles al hombre en sociedad para la definición de reglas de juego que le permitan interactuar y reducir la incertidumbre, en su relaciones categóricas de espacio-tiempo, naturalezapoblación-economía; con la característica de considerar la geopolítica como herramienta de poder que relativiza la soberanía en un mundo más comunicado e interdependiente y, en consecuencia, categóricamente proclive a la formación de instituciones políticas y económicas no inclusivas, donde la ciencia y la técnica ocupan un espacio preferente en la estructura del cambio económico, social, político y cultura de los Estados, es decir, que la desigualdad de los pueblos se explica entre otras razones, por las características de contexto de los países, así como por los cambios de la política económica diseñada en un entorno cambiante y fluctuante

Palabras claves: geopolítica, ciencia, desarrollo, tecnología, técnica, instituciones.

\section{abstract}

The document that one presents, departs from the recognition of the institutions as mental representations that are useful to man in society to define rules that allow you to interact and reduce uncertainty in space-categorical relationship time, nature-economy-population; with the feature of considering geopolitics as a tool of power relativized sovereignty in a world more connected and interdependent, and strongly inclined to the formation of political and non-inclusive economic institutions result, where science and technology occupy prominent space in the structure of economic, social, political and cultural change of states, ie inequality of peoples i explained among other things, the nature of country context, as well as changes as changes in economic policy designed in a changing environment and fluctuating.

Key words: Geopolitics, Science, Development, Technology, Engineering, Institutions.

\section{resumo}

O documento apresentado parte do reconhecimento das instituiçôes como representaçôes mentais que são úteis ao homem em sociedade para a definiçấo de regras de jogo que permitam interagir e reduzir a incerteza, em suas relaçóes

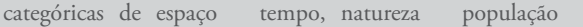
economia; com a característica de considerar a geopolítica como ferramenta de poder que relativiza a soberania em um mundo mais comunicado e interdependente e, em consequência, categoricamente inclinado à formaçáo de instituiçōes políticas e econômicas nâo inclusivas, onde a ciência e a técnica ocupam um espaço preferencial na estrutura da mudança econômica, social, política e cultural dos Estados, ou seja, que a desigualdade dos povos é explicada, entre outras razóes, pelas características de contexto dos países, assim como pelas mudanças da política econômica desenhada em um entorno mutável e flutuante.

Palavras-chave: geopolítica, ciência, desenvolvimento, técnica, instituiçōes

\section{Geopolítica, ciencia y tecnología en las sociedades con instituciones políticas y mercados no inclusivos}

\author{
LUIS ALFREDO MUÑNOZ VELASCO ${ }^{1}$ \\ JENNY L AVENDAÑO LÓPEZ ${ }^{2}$
}

\section{Introducción}

El propósito que gobierna este escrito, de carácter reflexivo, se centra en ubicar la concepción moderna del concepto de desarrollo en un contexto de geopolítica y, a partir de allí, con la ciencia, la tecnología y la técnica, en una clara referencia conceptual en su carácter instrumental a herramienta geopolítica, referir la existencia de sociedades con instituciones políticas y mercados no inclusivos (o extractivos), que explican la formulación de categorías de países y la existencia de desigualdades.

En esta perspectiva, la estructura de este documento pasa en una primera sesión por referir en un contexto histórico la geopolítica y los momentos de revolución industrial, como hitos que explican la forma y la esencia del mundo moderno en su contexto de relaciones de poder en el concierto internacional. En seguida, se aborda la geopolítica y el Estado, en una aproximación del ordenamiento espacial en función del papel del Estado en la definición de los mercados en una economía de corte capitalista. Por lo cual, se hace indispensable continuar con un aparte en el que se expone el efecto de causalidad que genera el progreso técnico junto con el conocimiento, sobre los niveles significativos de desarrollo. Finalmente, en un acercamiento conceptual de instituciones y organizaciones se aborda la referencia de sociedades con instituciones políticas y mercados extractivos (o no inclusivos). Luego se acoge el

1 Economista de la Universidad Santo Tomas, Bogotá; especialista en gestión del desarrollo regional, Universidad Surcolombiana, Neiva; especialista en administración financiera, Escuela de Administración de Negocios; especialista en docencia universitaria, Universidad Santo Tomas, Bogotá; especialista en seguridad Social, Universidad Externado de Colombia; magister en derecho económico, Universidad Externado de Colombia, Bogotá. Doctorando en desarrollo sostenible, Universidad de Manizales. Docente investigador, Facultad de Economía y Administración, Universidad Surcolombiana, NeivaHuila-Colombia. Luisalfredo.munoz@usco.edu.co

2 Economista Universidad Pedagógica y Tecnológica de Colombia; magister en ciencias económicas de la Universidad Nacional de Colombia. Docente investigador, Universidad Surcolombiana, Programa de Economía Jenny.avendanousco.edu.co 
desarrollo como expresión de libertad y, por último, se presentan algunas conclusiones.

Es así que el poder que materializa este intento académico está mediado por la necesidad de explorar en la teoría del desarrollo, y en particular la del desarrollo sostenible, argumentos que explican la existencia de diferenciales en las estructuras económicas y de poder que, desde lo político, hacen de los procesos de planeación de la acción, de las políticas públicas y el carácter de herramienta geopolítica de la ciencia, la tecnología y la técnica, un entramado que explica el progreso de la sociedad.

\section{La geopolítica y las revoluciones industriales}

En un enfoque conceptual e histórico, en ausencia de un determinismo característico de las posturas de los primeros teóricos a que hace alusión Huissoud \& Gauchon, (2013), es necesario ubicar la acepción geopolítica como referente, no solo de los cambios introducidos en la formación de la geografía política como espacio y tiempo, sino en las estructuras de poder que definieron la formación y surgimiento de relaciones de los Estados, y que explican la relación de fuerzas que han imperado e imperan aún, y que en el siglo XX, en una aproximación a la teoría de Spykman de un estado hegemónico (López, 2009) se conocen como de aislacionismo3 e intervencionismo 4 que, paradójicamente en una estructura global de las relaciones sociales de producción en un espacio cultural y humano mediado por cambios introducidos por la innovación y la ciencia, llevan a una visión del mundo en dos dimensiones categóricas de ricos y pobres, en la que la tecnología y la técnica han jugado un rol instrumental del desarrollo de los pueblos, pero también, la herramienta perfecta para el ordenamiento geopolítico del mundo.

O como dijera el licenciado en ciencias físicas de la Universidad de Buenos Aires, Rodríguez Trelles, "las ciencias y las tecnologias han perdido su

3 El aislacionismo, según the Free Dictionary, se define como la tendencia política que defiende el mantenimiento del país al margen de alianzas o conflictos internacionales.

4 Según la misma fuente el intervencionismo se define como tendencia política que defiende la intervención de un país en los asuntos internos de otro o sistema económico que defiende la intervención del Estado en la economía del país, en este caso se hace cargo de actividades de iniciativas privadas. carácter instrumental para convertirse en formidables herramientas geopoliticas"Ver vínculo Web Rodríguez, (2012). Luego, se han constituido en el vehículo sobre el cual explicar el éxito o fracaso de los países en una perspectiva de economías con instituciones políticas inclusivas y mercados inclusivos (Acemoglu \& Robinson, 2013), promotores de calidad de vida y bienestar entre los asociados o, economías caracterizadas por poseer y mantener instituciones políticas extractivas (no democráticas y excluyentes), con mercados a favor de dichas estructuras de poder, que los hacen extractivos (es decir no, inclusivos caracterizados por una mala distribución de la riqueza y una estructura a favor de unos pocos), responsables entonces, del atraso económico y de la miseria social de los pueblos.

$\mathrm{Y}$ es que, en este enfoque, la geopolítica se concibe como el "estudio de la influencia del espacio geográfico sobre los estados y su politica" (Cadena, 2006, p.117) y, en su dinámica evolutiva, no solo explica la formación de los Estados nación y su relación con la política, sino que se ha constituido en el eje de una política globalizada y de alianzas estratégicas en el transcurrir, que dan cuenta de los cambios económicos, sociales, políticos y culturales de los pueblos.

Es necesario indicar como los cambios de la humanidad han estado atados a tres hechos, que explican también los hechos políticos y los cambios económicos. Sin duda nos referimos a las tres hitos en que se han constituidos las revoluciones industriales que cuenta la historia.

Si ubicamos la primera revolución industrial en la Inglaterra de mitad del siglo XVIII (Lozano \& De la Cierva, 2004), daremos cuenta de cambios profundos o radicales en lo económico y poblacional. Antes de este hito, la economía se caracterizó por ser de naturaleza rural y artesanal; el uso de energías en los procesos productivos era humana o animal; además, en esta época, la región manifestaba bajos crecimientos demográficos, es decir que en el periodo preindustrial, el mundo, antes del descubrimiento de América, estaba dominado por la representación geográfica de Ptolomeo y de la visión cristiana regentada por la Iglesia católica que incluía a Europa, Asia y África. 
Después de la trayectoria heroica de Colón, y en el siglo XV, Martín Waldseemüller5 publicó un mapa del mundo [1507] que incluye el nombre de América, acompañado de la siguiente afirmación que recoge Nieto Ortega en la Geografía del nuevo mundo y el bautismo de América (2008), y que es quizás, un marco explicativo en el juego de poder en las relaciones internacionales y los determinantes del comportamiento de la política exterior de los gobiernos al indicar: "así como los granjeros señalan y dividen sus tierras con lineas fronterizas, ha sido nuestro propósito marcar los principales paises del mundo con los emblemas de sus gobernantes" (Nieto, 2008, p.5), sin duda una referencia tácita al propósito de la geografía política en la formación y delimitación de los Estados-nación y el ejercicio del poder político y económico, a partir del concepto de soberanía, definido por estructuras constitucionales y de derecho internacional, enfilado a lo que hoy se conoce como un concepto moderno de Estado supranacional, que soporta el surgimiento y los cambios paradigmáticos reflejados en el debilitamiento del concepto antiguo de Estados-nación, hacia una forma de organización propia del postconflicto y propia de la era del fin de la guerra fría, evolucionando hacia formas de organización política que revalúan el contenido de la soberanía en un entramado de relatividad convencional internacional, en el que la diplomacia y las armas "han cedido parte de su espacio a la economia, a la información, a la ciencia y tecnología" (Soto, 1996, p.31).

En esa referencia y en síntesis, durante el periodo que va de 1760 a 1830 , conocido como el tiempo de la primera revolución industrial, en el cual se destaca la transformación de la economía agraria con métodos artesanales de producción y el uso de energía humana y animal, a una economía denominada por la industria y la manufactura mecanizada, el uso de energía generada por el vapor de agua (Benavides, 2008) y el crecimiento demográfico; no solo es el comienzo de una gran transformación del concepto de dominancia económica, política y cultural, revolucionando entonces la visión y conceptualización aprendida del territorio y naturaleza, a uno que más adelante se conectaría con el concepto de espacio y hombre, en una simbiosis hombre-espacio-entorno, en el cual

5 Martin Waldseemüler, cosmographie introductio cum quibusdam geometriae a astronoiae principis ad eam rem necessaris, insuper quattour americi vespucci abigations, en : http://www.henry-davis. com/MAPS/Ren/Ren1/310morio.html no es posible concebir el espacio sin el hombre y a este sin un espacio determinado y definido por su entorno.

Luego, este juego de poder demarcado por la naturaleza y el hombre, viene necesariamente acompañado de expresiones culturales y políticas que dieron paso al surgimiento, también, de los derechos humanos de segunda generación (Rey \& Rodríguez, 2005), como una necesidad de reglas o instituciones propias de una convivencia entre clases surgidas a partir del nuevo orden económico imperante y un Estado más humano.

El segundo hito en la historia es sin duda el periodo definido como la segunda revolución industrial que, según Benavides, trascendió a Gran Bretaña y abarcó a Europa Continental y Estados Unidos, y se caracterizó por la "aplicación extensiva del conocimiento científico como principal insumo a la producción"(Benavides, 2008 p.60) o, lo que es lo mismo, la ciencia y la tecnología al servicio de la economía de producción. El uso de la energía eléctrica, el desarrollo de la química, los avances técnicos en la producción del hierro y el acero, el descubrimiento y uso de nuevos medios de transporte, el descubrimiento de nuevos insumos y materias primas, y la producción de nuevos productos $\mathrm{y}$ alimentos generaron la reducción de los costos y una noción de precios relativos y ventaja comparativa como condición de comercio en una economía de mercado, incentivando la promoción permanente de investigación y desarrollo en productos y mercados hacia el uso de nuevas tecnología y técnicas al servicio del nuevo sistema económico y político imperante en el orbe. Cabe resaltar que este periodo, que en un primer tramo se refiere a 1830 a 1850 , se caracterizó por un desarrollo complementario a las tecnologías existentes y que marcaron la primera revolución.

En el segundo tramo, que se ubica a partir de 1850, sobresalen los avances tecnológicos y la concentración de los procesos productivos en unidades más complejas. En este los sistemas de comunicación $y$ redes constituyen elementos significativos en la introducción de cambios paradigmáticos en la relación espacio-tiempo, política y poder, economía y sociedad, que se convierten en un escenario prolífero para el desarrollo teórico de las ciencias y, en particular, de una redefinición del Estado-soberanía, en un mundo hoy globalizado e interconectado en una clara connotación de sociedad red, "considerada como la del aplanamiento del mundo" (Friedman, 
2008, p.90) y, por tanto, una nueva diagramación del espacio y tiempo influenciada por la racionalidad económica y que ha llevado a una "homogenización de los patrones de producción y de consumo, contra una sustentabilidad planetaria fundada en la diversidad ecológica y cultural" (Leff, 2013. p 186) que, si bien ha llevado a cambios en la relación naturaleza-hombre, con impactos negativos sobre la primera, también es cierto que estos cambios han llevado a este último a reconsiderar su papel y responsabilidad en la vida diaria y la visión de futuro.

Entre tanto, Jeremy Rifkin, considera que "las grandes transformaciones económicas de la historia ocurren cuando una nueva tecnología en el campo de la comunicación converge con unos sistemas energéticos también novedosos" (Rifkin, 2011, p.57); a decir de este autor, la nueva tecnología de la comunicación es la internet, y la novedad en los sistemas energéticos es entonces la energía solar. La fusión de estas constituyen los elementos definitorios y argumentativos del surgimiento de la tercera revolución industrial que tiene sus inicios entre la última década del siglo XX y el comienzo del siglo XXI. Esta visión contemporánea, modifica la concepción de espacio y tiempo, volviéndola más dinámica en una elongación, no solo hacia y desde los mercados, sino a favor de un mundo socialmente más conectado, consciente e interdependiente, dueño de un futuro mejor, con lo cual la condición geoespacial, referida a una condición de ventaja en la dotación de recursos y producción limpias, se convierten en un atractivo y un potencial de negociación de poder en el nuevo mapa geopolítico, hacia donde tiende la redefinición del concepto de espacio vital, en esa visión de desarrollo sostenible que marca la nueva era del conocimiento.

\section{La geopolítica y el Estado}

Así las cosas, y parafraseando a Soto Gómez (1996), el análisis geopolítico es la herramienta de poder del gobernante de turno en el diseño de un futuro para su gobernados a partir de la definición de la identificación plena de la acción relacional hombreespacio-entorno-sociedad, dados unos condicionantes de carácter político, culturales, económicos y sociales que lo crean y lo definen.

Luego, el esfuerzo, del gobernante no es ya la formulación e implementación de políticas científicas y tecnológicas, en la que la "investigación básica condiciona de manera decisiva las oportunidades para una innovación tecnológica que, a su vez, determina el incremento de bienestar humano." (Gonzalez, López \& Lujan, 1996 p.8), es decir, la iniciativa no es del mercado, sino que es necesario orientar la acción investigativa en ciencia y tecnología que promuevan procesos transformativos en las estructuras de mercado y en la sociedad.

Entonces, recobra importancia para la acción gubernamental y para la comunidad científica, que $e l$ Estado asuma el liderazgo en el gasto en investigación y desarrollo - como porcentaje del PIB - aunque, en países como Argentina, Colombia, Chile, Ecuador y México no alcanzaba, en promedio, al $0,5 \%$ en el 2008; mientras que en Estados Unidos, España, Canadá, Reino Unido, Francia y Suiza, entre otros, superaba el 2,15\%, en promedio, en igual fecha. Ratios estos, muy por debajo del gasto del 3,5\% en Japón y del 3,4\% en Corea del Sur. Indicadores que se comportan similar en el 2010 (Banco Interamericano de Desarrollo, 2013), con lo cual, de una u otra forma, explican las teorías la dependencia y el atraso relativo existente en la geografía mundial y en la formación de alianzas estratégicas bajo el contenido de geopolítica.

Sin embargo, es importante resaltar aquí, la afirmación de León Olivé, en una apreciación de respuesta justa a la pregunta de si debe la sociedad, invertir más recursos en ciencia, a lo que responde:

La exigencia de una inversión cuantiosa en ciencia requiere una fundamentación en varios ejes, entre otros, desde el punto de vista ético, desde el económico, el político y sobre todo desde una perspectiva de justicia social entendida como la garantía de que todos los ciudadanos puedan satisfacer sus legítimas necesidades básicas, de acuerdo con la definición que los propios interesados hagan de esas necesidades, $\mathrm{y}$ por medios que les resulten aceptables según sus valores y formas de vida (Olivè, 2007, p.21).

Con lo cual, le revierte el carácter de instrumental al presupuesto público y, con ello, la exacerbación de las desigualdades sociales, dado el carácter subjetivo de justicia social aportado, y en ausencia clara de unos mínimos de progreso social que referencien y diferencien que, junto al elemento ético inmerso en la decisión, el cual constituye un peso significativo en las estructuras de poder y que en economías en las que el deseo de perpetuarse como sujetos indispensables a causas personales y electoreros, en un contexto 
de comunicación populista con sus gobernados, $y$ en contubernio con intereses de mercado, en específico a grupos económicos, le llevan a formular políticas que no necesariamente contienen ese ideal de respuesta que plantea Olivé, cargado de justicia social; en cambio sí, reflejando, tal vez como se puede observar en las políticas económicas y sociales impuestas no solo en América Latina y el Caribe, sino, en toda la geografía, un mayor número de pobres y excluidos, profundizando la brecha espacial y política, no solo al interior de las fronteras, sino entre países categorizados como pobres y ricos, aunque conectados en el ciberespacio pero excluidos de la economía, como dice Olivé en el prologo del libro El hombre y la técnica (2010).

Cabe anotar que esa subjetividad de justicia social entendida por Olivé, aunque enmarca un criterio de libertad centrada en valores y formas de vida en la elección de sus necesidades, no resuelve el ideal de justicia y progreso propio de la especie humana organizada en sociedad. En ese marco teórico, nos adentramos a una definición de desarrollo, característico de los pueblos y propio de las economías de mercado.

Acogemos aquí entonces, la afirmación que Amartya Sen hace de desarrollo, "como un proceso de expansión de las libertades reales de que disfrutan los individuos" (Sen, 2006, p.19), en la que la expresión del crecimiento económico, la industrialización, el progreso tecnológico, entre otros indicadores o herramientas para medir el cambio, son medios para la expansión de esas libertades y, en consecuencia, el fin u objetivo del desarrollo se centra en las libertades; las cuales tienen, en la clasificación de que de ellas hace Sen, una categoría instrumental que refuerzan los elementos integradores del progreso, en la que "las libertades politicas, los servicios económicos, las oportunidades sociales, la garantía de transparencia y la seguridad protectora" (Sen, 2006), son el eje fundamental que alimentan la concepción del desarrollo como libertad.

Hasta aquí el discurso planteado nos lleva retomar la apreciación categórica de Fisher, del ser técnico (2010), definido por la intencionalidad plena, la libertad y la opcionalidad como ejercicio de la razón y la racionalidad práctica eventual, que le dan soporte bajo el principio de tenacidad-intencionalidad y, en consecuencia, envuelve a la ciencia, la tecnología y la política, en instancias o manifestaciones concretas de la técnica, en las que su evaluación reviste un carácter público, es decir, del ámbito de la política, respecto a sus resultados frente a los individuos en cuanto a libertades y el ámbito de la sociedad, en lo que a justicia se refiere (Fisher, 2010). Para este autor la técnica es "un conjunto de elecciones y acciones orientadas por creencias y valores" (Fisher, 2010, 23) en una experiencia que él llama transaccional y que la ubica en el plano entre el hombre y su medio.

Es decir describe un "sistema técnico eficiente", donde el Estado se legitima en la medida que alcance esos ideales de libertad y justicia, en los cuales la ciencia y la tecnología juegan un papel importante; pero no es lo que dignifica y hace al hombre, sino que lo eleva a una dimensión de creador y dominador del medio a favor suyo y constructor social, en garantía de libertades y justicia social, que revitalizan y le dan sentido a la existencia del Estado y valor al ejercicio de la política, como acciones legitimadas.

La importancia conceptual referida cobra valor en la medida que el mundo se ha vuelto más proclive a la formación de bloques de países, con intereses comunes y que, aunque se conserva la independencia geográfica y se buscan formar alianzas regionales o acuerdos bilaterales, están motivados o los unen intereses económicos y políticos, para hacer frente a la globalización y el auge de las comunicaciones. Esta nueva formación de la geografía política ha conducido a relativizar el concepto mismo de soberanía a favor de una visión estratégica del espacio como extensión de poder político y mercado, en un esfuerzo común por alcanzar niveles de progreso y garantías de libertades entre los signantes; ejemplo de ello, son la zona Euro, Nafta, Mercosur y la Alianza Pacífica en construcción. Ahora, estos acuerdos comunes son responsables en gran parte de los resultados sociales, económicos que traducen en problemas como los que marcaron la agenda de los países miembros de Naciones Unidas y que se conocieron en el inicio del siglo XXI como Objetivos de Desarrollo del Milenio y que, aunque dan cuenta de que la pobreza extrema en el mundo, por citar uno de estos, se redujo a la mitad antes del año meta, (Programa de las Naciones Unidas para el Desarrollo, 2012), las desigualdades sigue siendo un elemento diferenciador y excluyente en gran parte del mundo civilizado. 


\section{La institución y los mercados inclusivos}

$\mathrm{Y}$ es que frente al problema desigualdades sociales, exclusión, instituciones populistas con mercados extractivos, la geopolítica, como referente instrumental para perpetuar diferencias regionales, entre otros $y$, que arriba se plantea, pareciera simplemente corresponder a una estructura del mercado y, en algunos casos, para satisfacer el interés de muchos inconformes que ven en las protestas sociales una oportunidad para confundir, obtener dividendos e indicar que los problemas sociales y económicos de la humanidad tienen como único responsable al modelo económico imperante y, por tal, el mercado, como responsable.

Sin embargo, las posiciones son divergentes. El premio nobel de economía Joseph Stiglizt, sobre este referente, señala que si bien el mercado contribuye a formar y crear las desigualdades, estas, son "consecuencias de las politicas de gobierno, tanto por lo que hacen como por lo que no hace" (Stiglitz, 2013, p.75), posición ideológica que aquí se expresa como hipótesis. El problema indica que, para alcanzar la reducción y/o desaparición de las desigualdades y alcanzar óptimos de justicia social, como elementos que legitiman al Estado, es necesario reescribir las reglas del juego o parafraseando a (North D. , 2006)6, la necesidad de que el hombre en sociedad fije límites a su actuación que le permitan dignificar su accionar interactivo como ser social, en el que la intencionalidad, la oportunidad y la racionalidad en la elección le lleven a un mejor vivir. Es decir tenemos que volver sobre las instituciones y aprovechar el cambio tecnológico, en su dimensión de "cambio en la relación entre insumos y producción" (Krugman, 2004, p.209), para la construcción de sociedades políticamente inclusivas en mercados más participativos con modelos claramente inclusivos, en los que el uso de la política económica busque cubrir a todos los sectores de la población.

En este contexto, las instituciones se constituyen para la sociedad en el mecanismo que permite "reducir la incertidumbre estableciendo una estructura estable

6 Para North (2006), las instituciones pueden ser formales e informales; las primeras son limitaciones impuestas por el hombre como la constitución política de un Estado; mientras que las segundas son acuerdos y códigos de conducta-evolucionadas. También se refiere a organizaciones que constituyen los jugadores en el marco de las estructuras de interacción humana, como son los partidos políticos y las universidades. (pero no necesariamente eficiente) de la interacción humana. Pero la estabilidad de las instituciones de ningún modo contradice el hecho de que estén en cambio permanente" (North, 2006. P. 16); indicativo de un continuo evolucionar de las instituciones que explica el cambio histórico vivido por la sociedad marcado por diferencias significativas entre países, lo que, $y$ siguiendo a Acemoglu y Robinson (2013), nos lleva a preguntarnos si las desigualdades existentes y que preocupan a los teóricos del desarrollo, economistas, sociólogos, entre otros, obedecen o son causadas por la condición geográfica, la cultura, o la ignorancia. Ello, nos lleva también a preguntarnos qué causa la creación de riqueza o prosperidad y pobreza en una sociedad.

Los autores arriba citados, y como lo demuestran en su libro Why Nations Fail, recurriendo a la historia y a escenarios geográficos como Corea del Norte y Corea del Sur, México antes de la llegada de los españoles, Nogales (Arizona-USA) y Nogales (Sonora-México), entre muchos otros desarrollos teóricos en perspectiva histórica, han concluido que, antes que las condiciones biofísicas de la región o el aspecto sociocultural o de inteligencia de los gobernantes y gobernados, que si bien tienen algo que ver con el estado de atraso y progreso de una sociedad, no son determinantes, los hechos que explican estas divergencias son de origen institucional (políticas) y, en consecuencia, determinadas por las organizaciones políticas que gobiernan el país. Una clara alusión a la política económica y sus políticas en el ejercicio del poder.

Es decir, que la presencia de instituciones políticas inclusivas en mercados inclusivos es responsable de la creación de la prosperidad en una sociedad determinada, dada la existencia de organizaciones que gobiernan con intereses distintos buscando el progreso de sus asociados. O sea, que son instituciones que:

Reparten el poder en la sociedad y limitan su ejercicio arbitrario. Estas instituciones políticas también dificultan que otras personas usurpen el poder y socaven las bases de las instituciones inclusivas. Quienes controlan el poder político no pueden utilizarlo fácilmente para establecer instituciones económicas extractivas en beneficio propio. Y estas instituciones económicas inclusivas, a su vez, crean un reparto más equitativo de los recursos, facilitando la persistencia de las instituciones políticas (Acemoglu \& Robinson, 2013, p.105). 
Hecho que no es una evidencia contundente en los países de América Latina y el Caribe, como tampoco lo es en un gran número de países que hoy reconocemos como pobres; sin que con esto estemos desconociendo que en los países ricos con instituciones políticas y mercados inclusivos no exista la desigualdad y pobreza. Solo que en estos las oportunidades que ofrecen estas condiciones de inclusión, coloca en mejores condiciones a los pobres frente a este mismo grupo en otras economía consideradas como subdesarrolladas.

Entre tanto, la pobreza de los pueblos se explica por la presencia de intereses distintos de los gobernantes que hacen de la política un instrumento para sacar provecho del ejercicio del poder encomendado, aliándose con sectores o grupos económicos que les permiten mutuamente conservar el poder y reelegir sus políticas, generando instituciones políticas extractivas y mercados extractivos (Acemoglu $\&$ Robinson, 2013). No basta un poder político estable y democrático para garantizar la presencia de instituciones inclusivas; pues, la existencia de grupos que generan conflictos internos, el deseo de perpetuarse en el poder7 limitando libertades, una política económica encaminada al aumento de coberturas en educación y salud, pero sin calidad; la limitación en el acceso a la ciencia y la tecnología, la presencia alta de niveles de corrupción, narcotráfico, entre otros, generan exclusión y, en consecuencia, desigualdades.

Mientras que una sociedad con instituciones políticas y económicas inclusivas, no solo hace respetar los derechos de propiedad, sino que crea igualdad de oportunidades, incentivos, fomento a la inversión y a la tecnología, los cuales se dan en el marco de gobiernos pluralistas y el reparto del poder, generando pesos y contrapesos. Lo anterior no quiere decir que en los países avanzados no haya pobres. Los hay y en un numero alto; pero, es de anotar que comparativamente con los pobres de países atrasados, ellos, tienen un nivel de vida e ingreso per cápita más alto y gozan de más oportunidades y libertades.

Ahora bien, los hechos históricos que han marcado el devenir de los pueblos y que aquí, hemos referenciados como los cambios derivados de las

7 Conservando el régimen político llamado democracia, introducen cambios en las constituciones políticas y convocan a elecciones, con el uso de estrategias electorales populistas. revoluciones industriales, en su destrucción creativa, han contribuido significativamente para explicar que, en aquellos países que hicieron de ellos un referente estratégico para introducir modelos de instituciones políticas y económicas inclusivas en beneficio de la sociedad, son hoy más prósperos.

En un mundo globalizado, el análisis de la geopolítica como herramienta de poder del gobernante en la definición de estructuras de poder caracterizadas por la acción relacional hombre-espacio-entornosociedad se constituye en un mecanismo acertado para reducir las brechas que existen entre países avanzados y atrasados, a partir de compartir no solo una relatividad convencional internacional de soberanía, mediada por mercados más incluyentes y competitivos, sino también permitiendo el acceso a los resultados de la ciencia, la tecnología y la técnica (como habilidad), y apoyando la formación de alto nivel de estructuras educativas y políticas que promueven la formación de instituciones políticas y económicas inclusivas.

\section{El desarrollo en la sociedad moderna del conocimiento}

El desarrollo económico en las sociedades modernas ha experimentado cambios contundentes y súbitos (Adelman, 2002). El periodo de la Gran Depresión, y posterior suceso de la Segunda Guerra Mundial, dejaron presumir al interior de la corriente del pensamiento económico la profunda preocupación o inquietud en torno a los procesos de desarrollo de los países con menos avance en lo económico o social (subdesarrollados o países en desarrollo), siendo la política un elemento integrador de decisiones, acorde a las condiciones de cada economía.

En correspondencia con las disposiciones de política, relacionadas con el grado de intervención del gobierno, la naturaleza de la interacción de este y el mercado ha estado determinada por las prescripciones derivadas del modelo de desarrollo. Para Adelman, los cambios en estos direccionamientos de lo político, a lo largo de la historia, se vinculan a la transformación de la dinámica en varios escenarios, como el aprendizaje, las ideologías alternativas, evolución en el entorno internacional y cambios en las instituciones (2002); sin otorgar un lugar primordial a ninguna variable como la responsable de las tendencias del desarrollo, al unísono y conforme avanza la modernidad, son elementos como el capital físico, el espíritu 
empresarial, el comercio internacional, la modernidad del Estado, pero sobre todo el capital humano, los que determinan la dinámica y los niveles de desarrollo económico.

Entonces, un elemento de relevancia y diferenciador en el desarrollo confluye en la idea de la importancia del capital humano y el influjo de la innovación; postura que en las corrientes del pensamiento económico se han mantenido con el paso del tiempo "aunque la naturaleza está sujeta a rendimientos decrecientes, el hombre está sujeto a rendimientos crecientes (...), el conocimiento es el combustible más poderoso de la producción; éste nos permite dominar la naturaleza y satisfacer nuestros deseos" (Marshall, 1890. En: Meier, 2001; p 8 ). Un siglo después, Romer redescubre la importancia del progreso técnico determinado por la acumulación continua del conocimiento de los mismos maximizadores de los beneficios (1986). El progreso técnico y la formación de capital humano son endogeneizados al interior de modelos de crecimiento de equilibrio general. El nuevo conocimiento es forjado gracias a la inversión en el sector de investigación, y el progreso técnico residual es contabilizado mediante la formación endógena de capital humano y los incrementos en el almacenamiento público de conocimiento (Meier, 2001).

\section{El desarrollo como expresión de libertad o la libertad expresada en el desarrollo}

Al responder a la pregunta, desarrollada en una entrevista en Chicago Tribune, sobre qué se considera una economía exitosa, el economista, Sen, argumentó que la posibilidad que los miembros de una sociedad, sobre todo los que se encuentran en peores condiciones, tengan de participar en la elección de las decisiones y oportunidades que mejoren su bienestar, y así mismo el abandono por parte del Estado de la población que se encuentra en la escala inferior, evidenciará una economía fracasada (Sen, 1999).

Para Sen, el desarrollo es entendido como "un proceso de expansión de las libertades fundamentales que tienen los individuos"(1999, p.355), en el que la existencia de instituciones y organizaciones ayudan y contribuyen a mantener y aumentar las libertades individuales. En el entendido para Sen, las libertades involucran "procesos de toma de decisiones como oportunidades para lograr resultados valorados” (1999, p.348), y el desarrollo no es solo en una visión reduccionista de crecimiento económico, en el que el capital humano y capacitado representa un factor determinante para aumentar la productividad, sino que es necesario observar a los seres humanos en una dimensión que comprende un universo más complejo como es el de las capacidades humanas, como la libertad de vivir la vida que mejor les parezca a partir de una formación educativa que les permita elegir libremente, con la cual se constituyen en influenciadores del bienestar social y de las libertades individuales, en el cambio social y económico.

Luego, la garantía de más libertad obliga al hombre a ser más responsable no solo de sí mismo, sino en su relación entorno-sociedad hacia una comprensión de ser técnico. En este contexto, podemos considerar "el desarrollo sostenible como la satisfacción de las necesidades de la generación presente sin comprometer la capacidad de las generaciones futuras para satisfacer sus propias necesidades". (ONU, 1987, p.60), con lo cual, no solo se ha marcado el camino para estructurar políticas que permitan alcanzar el desarrollo en lo económico, social, sino también en la necesidad mundial de protección del medio ambiente, es decir, la técnica; con lo cual la geopolítica, como herramienta de poder, se constituye y empodera en un aliado del medio ambiente y, por consiguiente, de la promoción de la ciencia y la tecnología hacia la concepción de un mundo garantizado para las generaciones actuales y futuras bajo un concepto de Estado valorizado y legitimado como ente que responde a un constructo social, es decir, un sistema técnico eficiente.

\section{Conclusiones}

Las teorías del desarrollo promovidas por economistas, sociólogos, antropólogos, politólogos, ven como explicación de los niveles de atraso y/o subdesarrollo de las economías de África, Asia, América Latina y el Caribe elementos explicativos derivados de las condiciones geográficas, el clima, la cultura, los aspectos sociodemográficos, entre otros; sin embargo, no cabe duda que el nivel de atraso de algunas economías, no solo obedecen al desaprovechamiento histórico de las oportunidades que los cambios tecnológicos y técnicos propias de las revoluciones industriales les ofrecieron, sino, a la acción y/o inacción de los gobernantes en la aplicación de políticas en un contexto de instituciones políticas y económicas no inclusivas, antes que las mismas condiciones de la geografía política. 
De otra parte, la visión de la tecnología no solo entendida como ciencia aplicada, sino como cambio tecnológico que explica la evolución y el crecimiento, manifiesta en gran medida el mapa geopolítico y, por ende, la formación y avance hacia una consolidación de países avanzados y/o desarrollados, con lo cual se da cuenta de las brechas existentes con las economías que no han sabido aprovechar las revoluciones que el mundo a vivido y que, en los últimos años, han sido proliferas y el escenario perfecto para repensar el país hacia un modelo inclusivo.

Hoy, cuando el mundo se mueve en torno a una tercera revolución industrial que los expertos definen y sustentan en las comunicaciones vía internet y la generación de energías alternativas, como la solar y eólica, en una clara connotación de sociedad red, es importante señalar que los países pobres, pero referenciados de gran potencial en recursos vitales para la humanidad del siglo XXI, encuentran en esta una oportunidad protagónica en las nuevas relaciones de poder que define la geopolítica mundial y, con ello, la posibilidad ofrecerle a su pueblo instituciones políticas y económicas inclusivas, en las que la libertad, como expresión del desarrollo, es garantía de un vividero mejor para nosotros y nuestros hijos en el presente y futuro.

Luego, la capacidad para permitir la destrucción creativa, tanto en el campo de la ciencia, la tecnología, la técnica como en las estructuras de poder, que permitan la definición de reglas que lleven a la consolidación de instituciones políticas y económicas que garanticen el pluralismo y la justicia, fundados en una educación incluyente y de calidad con acceso universal en toda la estructura curricular, desde el preescolar hasta la universitaria y de postgrados, consciente de una visión sistema de investigación y desarrollo científico, romperá el circulo vicioso de la pobreza y la desigualdad, con ello, se dará la garantía de la evolución y progreso social, bajo el paradigma de un Estado legitimado y eficiente. 
Acemoglu \& Robinson, D. \&. (2013). Los origenes del poder, la prosperidad y la pobreza (tercera ed.). (M. M. Garcia, Trad.) Bogotà, D.C, Colombia: Planeta Colombiana S.A.

Adelman, I. (2002). Falacias en la teoría del desarrollo y sus implicaciones en política. En J. Stiglitz, Las fronteras de la economía del desarrollo (págs. 112 - 130). BID.

Banco Mundial. (2013). Gasto en investigaciòn y desarrollo (\% del PIB). Recuperado el 09 de 08 de 2013, de http//www.datos. bancomundial.org/indicador/6B. XPD.RSDV.GD.ZS

Benavides, Ô. A. (2008). De inventores a empresas-La historia econòmica de la innovaciòn tecnològica (Primera ediciòn ed.). (U. E. Colombia, Ed.) Bogota, D.C, Colombia: publicaciones @ uexternado.edu.co.

Cadena, J. L. (enero-junio de 2006). La geopolitica y los delirios imperiales de la expansiòn territorial a la conquista de mercados. Revista de relaciones internacionales, estrategia $y$ seguridad(1), 115-141.

Douglass, N. C. (2006). Instituciones, cambio institucional $y$ desempeño econòmico (tercera ediciòn ed.). (A. Bàrcena, Trad.) Mèxico, D.F., Mèxico: Fondo de cultura econòmica.

Fisher, J. (2010). El Hombre y la Tècnica, Hacia una filosofia politica de la ciencia y la tecnologia. (U. N. Mèxico, Ed.) Mèxico, D.F., Mèxico: Socieda y cultura, Mexico Siglo XXI.
Friedman, T. (2008). La tierra es plana (tercera ediciòn ed.). Bogotà, D.C., Colombia: Planeta colombiana S.A.

Gonzalez \& Lòpez \& Lujan, M. I. (s.f.). Los estudios sobre ciencia, tecnologìa $\mathrm{y}$ sociedad. En S. Fuller, La Ciencia, La Tecnologia y La Sociedad.

Huissoud \& Gauchon, J. M. (2013). Las 100 pañabras de la geopolitca. (2. Presses universitaires de france, Ed.) Madrid, España: ediciones Akal.

Krugman, P. R. (2004). Internacionalismo POP. Bogotà, D.C, Colombia: Grupo Editorial Norma.

Leff, E. (abril de 2013). La geopolitica de la biodiversidad y el desarrollo sustentable. Economizaciòn del mundo, racionalidad ambiental $\mathrm{y}$ reapropiaciòn social de la naturaleza. Cuaderno interdisciplinar de desarrollo sostenible(10), 185-209.

Lòpez, J. I. (0707 de 2009). La Geopolitica de Nicolas Spykman. Revista Universidad Eafit(97-100), 79-86.

Lozano \& De la Cierva, J. J. (2004). Revista digital de historia y ciencia sociales. Recuperado el 01 de 08 de 2013, de Claseshistoria. com: http://www.claseshistoria. $\mathrm{com} /$ revoluciònindustrial/1 revolu cionindustrial.html.

Marshall, A. (1890). Principles of Economics. Library of economics and liberty.

Meier , G. (2001). The older generation of economists desarrolllo and new . In J. Stiglitz,
Economics frontier development. $\mathrm{Sl}$ : Inter-American Development Bank

Nieto, M. O. $(13$ de 06 de 2008). Historia Natural y Politica: conocimientos y representaciones de la naturaleza amèricana. Recuperado el 03 de 08 de 2013, de Bibllioteca Luis Angel Arango-Banco de la Repùblica: www.banrepcultural. org/blaavirtual/exhibiciones/ historia..../hnp-04.htm

Olivè, L. (2007). La ciencia y la tecnologia en la sociedad del conocimiento. Etica, politica y epistemologia. (primera ediciòn ed.). Mexico, D.F, Mexico: Fondo de Cultura Econòmica.

ONU. (1987). Informe Bruntland Agosto (1987). Nuestro Futuro Comùn. Comisiòn mundial sobre medio ambiente y el desarrollo. Documentos oficiales de la Asamblea General, Cuadragésimo Segundo periódo de sesiones, Suplemento No 25 (A/42/25)

PNUD. (2012). Naciones Unidas. Recuperado el 12 de 08 de 2013, de http://www.undp.org/content/ undp/es/home/librarypage/mdg/ the-millennium-developmentgoais-report-2012/

Rey, \&. R. (2005). Las Generaciones de los Derechos humanos (Tercera Ediciòn ed.). Bogotà, D.C., Colombia: Universiadad Libre.

Rifkin, J. (2011). LA tercera Revoluciòn Industrial. (S. Espasa Libros, Ed., \& A. M. Santos, Trad.) Barcelona, España: Paidòs.

Rodríguez Trelles, Félix . (2012). CIENCIA Y TECNOLOGÍA: informe de situación $y$ 
perspectivas. 8 DICEMBRE Journal of Political Economy, 02-

2014, de Análisis Geopolítico 37. desde Suramérica Sitio web: http://www.dossiergeopolitico. com / $2012 / 06 /$ ciencia-ytecnologia-informe-de-situaciony-perspectivas.html

Romer, P. (1986 ). "Increasing Returns and Long - Run Growth.
Sen, A. (28 de Marzo de 1999). (C. Tribune, Entrevistador)

Sen, A. (2006). Desarrollo y Libertad. Bogotà, D.C., Colombia: Editorial Planeta colombiana S.A.
Soto, M. G. (1996). Hacia una nueva visiòn. (R. F. Econòmicas, Ed.) Investigaciòn \& Desarrollo Social, 16(16), 31.

Stiglitz, J. E. (2013). El Precio de la Desaigualdad. (segunda ed.). (A. Pradera, Trad.) Bogotà, D.C., Colombia: Taurus. 
